\title{
Imaging and non-imaging illumination of DLP for high resolution headlamps
}

Ley, Peer-Phillip, Lachmayer, Roland

Peer-Phillip Ley, Roland Lachmayer, "Imaging and non-imaging illumination of DLP for high resolution headlamps," Proc. SPIE 10932, Emerging Digital Micromirror Device Based Systems and Applications XI, 1093200 (4 March 2019); doi: $10.1117 / 12.2507436$

SPIE. Event: SPIE OPTO, 2019, San Francisco, California, United States 


\title{
Imaging and Non-imaging Illumination of a DLP for High-Resolution Headlamps
}

\author{
Peer-Phillip Ley and Roland Lachmayer ${ }^{\mathrm{a}}$ \\ ${ }^{a}$ Leibniz Universität Hannover, Institute of Product Development, Welfengarten 1A, Hannover, \\ Germany
}

\begin{abstract}
Matrix-LED systems offer different functionalities to increase road safety, e.g. glare-free high beam and marking light. Shortly after their introduction, efforts have been made to increase the amount of pixels. One of the results is the EVIYOS LED consisting of 1024 individually controllable pixels, which practically set the stage for pixel light systems. Current efforts to implement high-resolution pixel light systems are focused towards the exploration of an efficient light source in combination with the use of spatial light modulators.

One approach to implement high-resolution pixel light systems is the use of LED arrays as a light source to illuminate a DLP. Unlike video projectors which require a homogeneous illumination of the DLP in order to obtain a homogeneous projection, headlamps require an inhomogeneous light distribution with high illuminance in the center. In order to receive a high system efficiency preforming the desired illuminance onto the active area of the modulator is advantageous. To further increase the systems efficiency an imaging illumination of the DLP, where the images of the emission surfaces of the LEDs are superposed onto the active area of the DLP, is worthwhile.

In this paper, concepts for imaging and non-imaging illumination strategies of a DLP for high resolution headlamps will be introduced. For both illumination strategies the most promising concept will be selected to set up an optical system to illuminate a DLP. The paper concludes with a comparative analysis of the imaging and non-imaging optical system with regards to the system architecture and system efficiency.
\end{abstract}

Keywords: High Resolution Headlamps, DLP, Imaging Illumination, Non-imaging Illumination, LED

\section{INTRODUCTION}

In the past decades, a change in the field of vehicle lighting technology occurred with regards to efficiency and lighting functions, starting with the introduction of adaptive front lighting systems (AFS) in 2006. Using gas discharge lamps as light source, these systems enable the implementation of different dynamic light distributions such as town light, motorway light, bending light as well as low beam (Figure 1 (a)) and high beam (Figure 1 (b)) through the use of a special roller. By rotating the roller, which acts as an adaptive aperture within the ray path, the desired light function can be realized. The contour of the roller is imaged by a projection optics into the traffic area. By developing more efficient and brighter LEDs, the next step was the implementation of full-LED headlamps. In 2010 the first full-LED headlamp with AFS-functions was introduced in the Audi A8. These headlamps are more efficient, due to the opportunity to switch single LEDs on or off. At the same time they allow an individual adaptation of different light functions.

With high-resolution headlamps, the extent of implementable light functions will increase in the future. The light distribution of these headlamps consists of a large amount of pixels, similar to projectors in the entertainment

Further author information: (Send correspondence to P.-P. Ley.)

P.-P. Ley: E-mail: ley@ipeg.uni-hannover.de, Telephone: +49 5117624591

Emerging Digital Micromirror Device Based Systems and Applications XI, edited by

Michael R. Douglass, John Ehmke, Benjamin L. Lee, Proc. of SPIE Vol. 10932, 1093200

(C) 2019 SPIE · CCC code: $0277-786 X / 19 / \$ 18 \cdot$ doi: $10.1117 / 12.2507436$

Proc. of SPIE Vol. 10932 1093200-1 
industry. Depending on the technology (LCD, LCoS, DLP) applied to generate the image and the optical system, a variable amount of pixels can be realized. While with a small amount of pixels, as with the use of LED arrays, an individual illumination of the traffic area can be implemented, more pixels additionally enable the projection of information onto the road.

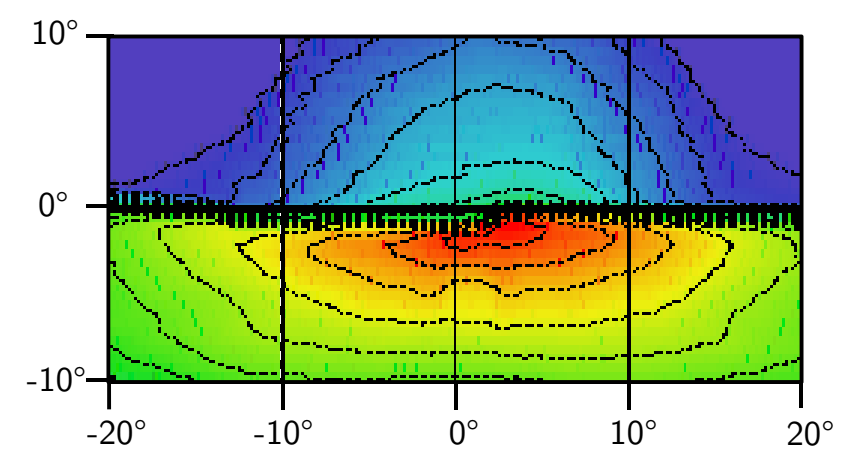

(a) Low Beam

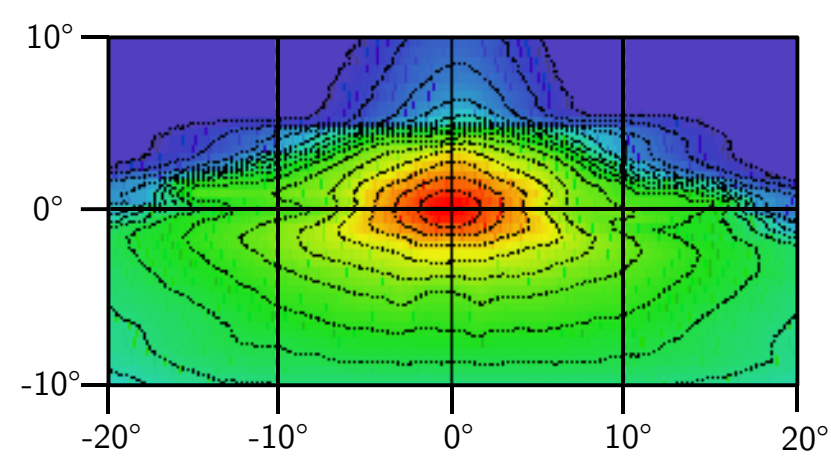

(b) High Beam

Figure 1. Cutouts of a low beam (a) and high beam (b) light distribution of motor vehicles on a wall 25 meters away

The technologies mentioned are subtractive imagers in which unused light is either reflected into an absorber (DLP) or blocked by liquid crystals (LCD or LCoS). In the field of video and entertainment, where the focus is less on efficiency but more on the dynamic and individual projection of images, absorbing or blocking light is less of an issue. However, in the field of vehicle lighting technology efficiency is a crucial factor in the development of lighting systems. Suitable illumination concepts, such as an inhomogeneous illumination of the active area of the modulator instead of a homogeneous, allow to increase the efficiency of these systems. ${ }^{1}$ The implementation of different illumination concepts is particularly dependent on the optical system to illuminate the active area of the modulator and the optical system for projection, as well as the technical implementation of the arrangement of these two optical systems.

\section{SYSTEM ARCHITECTURE AND TECHNICAL IMPLEMENTATION}

Unlike LCDs, DLPs are reflective spatial light modulators. Accordingly, illumination path and projection path share the same space in front of the modulator. The system architecture deals with the ray path of the optical system and makes the implementation to separate the illumination path and projection path a subject of discussion. Basically, when using a DLP as a spatial light modulator, a distinction can be made between a telecentric and a non-telecentric system architecture. ${ }^{2}$

The telecentry of optical systems results from the position of the aperture stop within the optical system. Multiple lens systems exhibit an object space telecentry when the aperture stop is located at the focal point of the front lens group. The path of the chief rays in object space is parallel to the axis and the entrance pupil is located at infinity in image space. The exit pupil is embedded in the optical system. An image space telecentry can be achieved by shifting the aperture stop to the focal point of the rear lens group, resulting in an axially parallel path of the chief rays in image space. The exit pupil is located at infinity in object space and the entrance pupil is embedded in the optical system. An optical system with object space and image space telecentry results from an aperture stop located at the intersection of the respective focal lengths of the front and rear lens group. Both the entrance pupil (in image space) and the exit pupil (in object space) are located at infinity. In a non-telecentric architecture, the exit pupil of the illumination optics is located at short distance from the active area of the modulator, by which means the entrance pupil of the projection optics must be coincident. The converging rays lead to different angles of incidence of the chief rays on the single micromirrors within the array. 
The technical implementation of the respective architecture can be achieved differently when using a DLP. However, both architectures have in common that the incident light on the active area of the modulator has to exhibit an angle which corresponds to the tilt angle of the micromirrors. For telecentric systems, the application of a TIR prism (Figure 2 (a)) or reverse TIR prism is suitable. All chief rays are parallel when they impinge on the micromirrors of the active area of the modulator. The technical implementation of a non-telecentric architecture differs from that for the telecentric architecture, since the entrance pupil of the projection optics and the exit pupil of the illumination optics must coincide. To achieve that freeform mirrors and folding mirrors can be applied within the ray path. Another way is to increase the illumination angle onto the DLP, allowing greater angular separation of the illumination path and the projection path (Figure 2 (b)). The greater separation of the two light paths makes it possible, depending on the size of the light source, to eliminate freeform mirrors and folding mirrors. ${ }^{3}$ At the same time, increasing the illumination angle leads to a higher overall contrast, but also to a reduction in light throughput. ${ }^{2}$ In addition to that the application of a field lens along the optical axis of the projection optics is required.

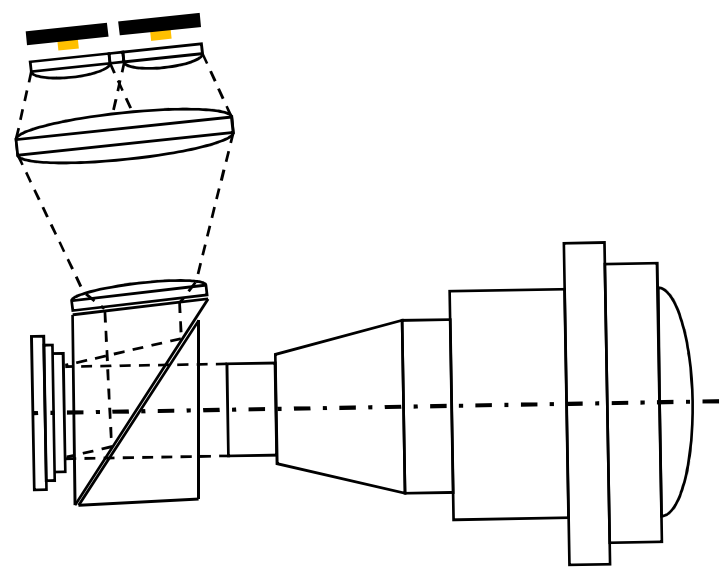

(a) Telecentric implementation using TIR-Prism

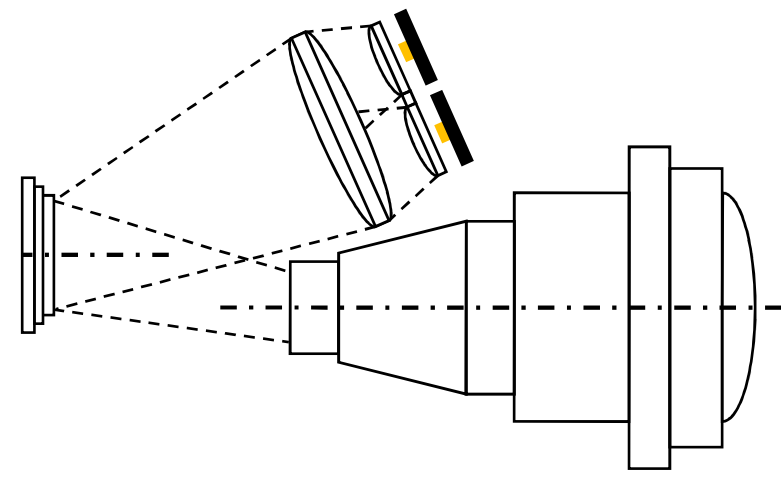

(b) Non-telecentric implementation by tilting the DLP

Figure 2. Technical implementation of the different system architectures

Each of these architectures and their technical implementation shown in Figure 2 has different advantages and disadvantages, which are presented below in excerpts in Table 1.

Table 1. Advantages and disadvantages of the system architectures and their technical implementation shown in Figure $2^{2}$

\begin{tabular}{|l|l|l|l|}
\hline \multicolumn{2}{|c|}{ Telecentric } & \multicolumn{1}{c|}{ Nontelecentric } \\
\hline \multicolumn{1}{|c|}{ Advantage } & \multicolumn{1}{|c|}{ Disadvantage } & \multicolumn{1}{c|}{ Advantage } & Disadvantage \\
\hline $\begin{array}{l}\text { Less distortion when illumi- } \\
\text { nating the modulator }\end{array}$ & $\begin{array}{l}\text { Polarization effects at the } \\
\text { air gap of the two prism } \\
\text { halves }\end{array}$ & $\begin{array}{l}\text { First lens of the projection } \\
\text { optics can be smaller due to } \\
\text { the finite pupil location }\end{array}$ & $\begin{array}{l}\text { The incident light onto the } \\
\text { modulator exhibits nonuni- } \\
\text { form angles }\end{array}$ \\
\hline $\begin{array}{l}\text { Independent design of illu- } \\
\text { mination and projection op- } \\
\text { tics }\end{array}$ & $\begin{array}{l}\text { Losses at the air gap of the } \\
\text { two prism halves of 2-3\% } \\
\text { per surface }\end{array}$ & $\begin{array}{l}\text { Higher overall contrast } \\
\text { compared to telecentric } \\
\text { design }\end{array}$ & $\begin{array}{l}\text { lower overall light through- } \\
\text { put due to the increased } \\
\text { illumination angle of the } \\
\text { DLP }\end{array}$ \\
\hline
\end{tabular}

Depending on the illumination concept, the respective advantages and disadvantages of the two architectures have different effects on the implementation of vehicle headlamps. For example, a high contrast is advantageous for the projection of information into the traffic area. In a non-telecentric architecture the maximum overall contrast is higher than in the telecentric architecture. However, at the same time a high light throughput is importent to implement a highly efficient system. Nevertheless, due to the increase of the illumination angle of 
the DLP (Figure 2 (b)), the non-telecentric architecture leads to a reduced light throughput compared to the telecentric architecture utilizing a TIR prism. These and other advantages respectively disadvantages are being taken up in Section 3 and discussed with regard to their suitability with the introduced illumination concepts.

\section{ILLUMINATION CONCEPTS FOR A DLP}

As already mentioned in the introduction, except for the technical implementation to illuminate the spatial light modulator, the field of application make further demands on the implementation of high-efficient systems. One of these requirements is the precise illumination of the active area of the DLP, for which various concepts are conceivable. In Table 2 drafts of selected concepts for an imaging and non-imaging illumination are illustrated. The draft of concept of the illuminance on the modulator surface are classified for better understanding. Red areas indicate high illuminance and blue areas indicate low illuminance.

Imaging concepts pursue the approach that through superposition of the single images of the emission surface of the light source, in this application LEDs, an advantageous distribution of illuminance results on the active area of the modulator. With regard to the image ratio, whether it is constant or individual, the imaging illumination concepts can be differentiated. In case of a constant image ratio the single images of the emission surfaces on the active area of the modulator exhibit the same size (Figure 3). On the other hand, an individual image ratio leads to images of the emission surfaces on the active area of the modulator of different sizes.

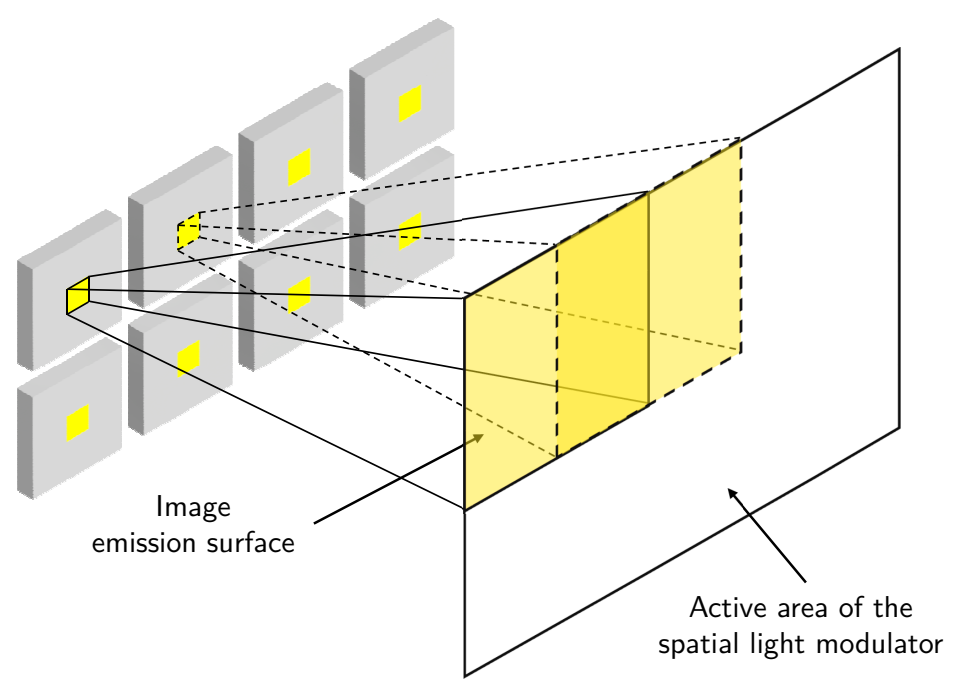

Figure 3. Superposition of the images of the emission surfaces of LEDs onto the active area of the spatial light modulator

Non-imaging illumination concepts can be divided into homogeneous and inhomogeneous. In the case of a nonimaging homogeneous illumination, the illuminance is constant over the entire active area of the modulator. This is e.g. required for video projectors. In the field of vehicle lighting technology, which requires inhomogeneous light distributions in the traffic area, as shown in Figure 1, this illumination concept is less suitable. Challenging in the generation of an inhomogeneous non-imaging illumination of the rectangular modulator surface is the combination of rotationally symmetric lenses and the mostly square emission surfaces of the LEDs, due to the resulting overfill. This overfill leads to a reduction in system efficiency, since some of the light illuminates the spatial light modulator outside of the active area.

From a conceptual point of view, particularly imaging illumination concepts are suitable since the system efficiency can be increased by switching off single LEDs of unused areas of the active area of the modulator, e.g. for a low beam distribution. 
Table 2. Illumination concepts for spatial light modulators ${ }^{4}$

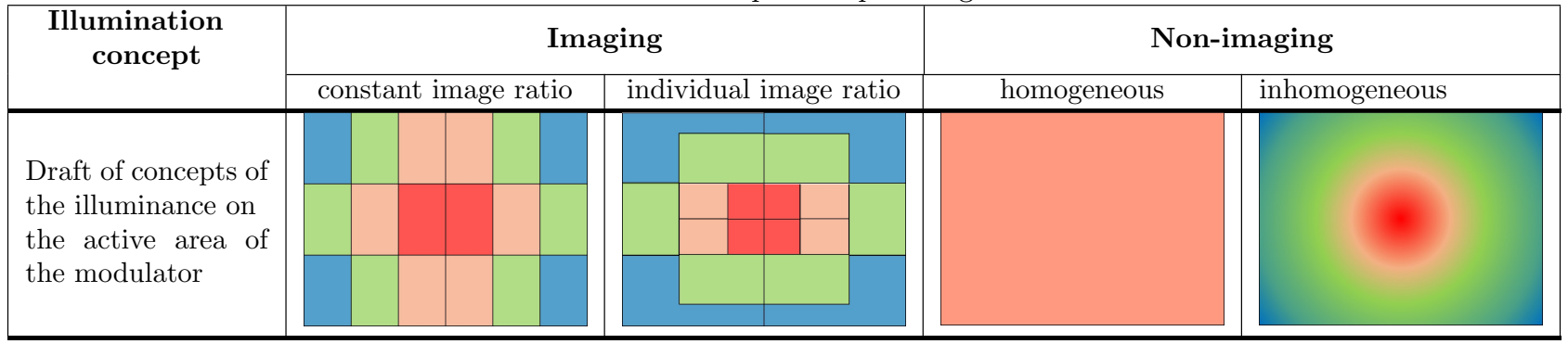

The imaging illumination concept with constant image ratio, in which eight equally sized images are superposed, allows the multiple use of one imaging optical system for each of the eight LEDs within the LED array. Therefore the use of different optical systems within the LED array is not required, as in the case of concept 2 with individual image ratio. However, imaging illumination concepts have a high sensitivity with respect to geometrical deviation of optical elements ${ }^{4}$ and position deviations. For this reason, particularly the telecentric architecture for the implementation of imaging illumination concepts is interesting, since a displacement of the light source (object space) along the optical axis ideally has no or only minor impacts on the image size (image space) (Table 1). However, when using a non-telecentric architecture for the optical system, a displacement of the light source in object space along the optical axis results in different image sizes on the active area of the modulator in image space. An incorrect positioning along the optical axis accordingly has a negative impact on the system efficiency. The reason for this is the same effect, the overfill, which also occurs when applying a non-rotationally symmetrical lens for an inhomogeneous non-imaging illumination. In addition to that, an incorrect positioning leads to distorted images of the emission surfaces in the traffic area.

Challenging in the technical implementation of an imaging illumination concept for a telecentric system architecture is the application of a TIR prism for the separation of the illumination path and projection path. In order to be able to superpose the individual images of the LEDs onto the small area of the spatial light modulator, an angular arrangement of the single LEDs and optics is required, which is shown for concept 1 in Figure 4 . For a clear illustration, only one row of the $2 \times 4$ array with two LEDs $(2,4)$ is shown. For each of the two LEDs, the chief rays are considered which impinge at different angles $\left(\theta_{2}, \theta_{4}\right)$ on the interface of the two prism halves.

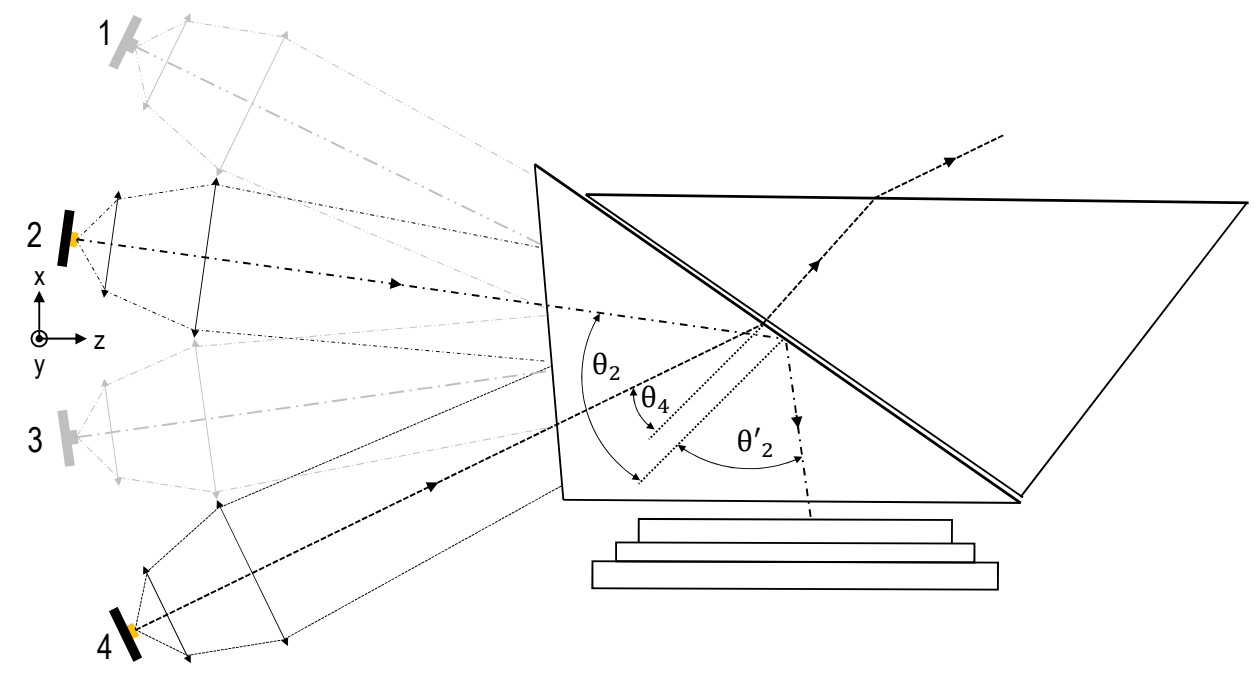

Figure 4. X-z plane of the three-dimensional LED array for an imaging illumination of the DLP using a TIR prism. 
This angular arrangement implicates a three-dimensional LED array and different angles of incidence at the interface of the two prism halves. Usually NBK-7 is applied as the material for TIR prisms, which exhibits a refractive index of $n_{1}=1.515$ at $632,8 \mathrm{~nm} .{ }^{5}$ The refractive index of air is $n_{2}=1$. The angle at the interface of the two prism halves under which total reflection occurs can thus be determined according to equation 1 , the Snell's law of refraction.

$$
\theta_{T I R}=\arcsin \left(\frac{n_{1}}{n_{2}}\right)=\arcsin \left(\frac{1,515}{1}\right)=40,3^{\circ}
$$

Rays which impinge on the interface of the two prism halves at an angle less than $\theta_{T I R}$, such as $\theta_{4}$, do not undergo total reflection. Consequently, the rays are not reflected onto the active area of the modulator but, after multiple refraction pass out from the prism or undergo total reflection at other interfaces of the prism. Since these rays act as scattered radiation in the system, they have a negative impact on the contrast, both on the modulator surface and on the projection in the traffic area. In addition, the scattered radiation is unused light, therefore reducing the system efficiency.

Using numerical simulations (Figure 5) it is possible to illustrate the impact of the TIR prism within the ray path on the imaging quality when superposing the single emission surfaces of the LEDs onto the active area of light modulator. Figure 5 (a) represents the ideal case for superposing the emission surfaces of 8 Osram LUW HWQP LEDs on the active area $\left(14.00 \cdot 10.50 \mathrm{~mm}^{2}\right)$ of a DLP7000 ${ }^{6}$ and corresponds to the concept with constant image ratio in Table 2. The distribution of the illuminance on the light modulator proves to be advantageous for a high beam distribution. For a low beam distribution, only half as much luminous flux and a lower illuminance in the traffic area is required. Switching off a complete row, 4 LEDs, of the LED array (Figure 5 (b)), significantly increases the system efficiency. The TIR prism in the ray path leads to magnified and out of focus images of the single emission surfaces on the active area of the light modulator, resulting in an overfill (Figure 5 (c)). Switching off one row of the LED array also does not increase the system efficiency, since almost the entire active area of the modulator is still being illuminated (Figure 5 (d)). With this setup, not only the system efficiency is not increased, but much more decreased, when implementing a low beam distribution. One approach to improve the imaging quality on the active area of the modulator would be to make the optics of the LEDs smaller. This can reduce spacing between the LEDs within the array, ${ }^{7}$ leading to more advantageous angles of incidence at the interface of the two prism halves. Another possibility is to reduce the amount of LEDs, which, however, means that the implementation of the illumination concept cannot be realized. However, both approaches result in a system with reduced light throughput, because either smaller optics or fewer LEDs are applied.

For the present application, therefore, a non-imaging inhomogeneous illumination of the DLP seems to be suitable. In a non-telecentric system architecture an inhomogeneous illumination of the active area of the modulator can be achieved due to an increased illumination angle. By applying a suitable projection optics, it is possible to achieve an illumination of the traffic area which is advantageous for vehicle headlamps. However, the intended separation between the illumination path and projection path can lead to an insufficient separation when using a large light source, such as an LED array. Consequently, a part of the light reflected from the DLP into the projection optics is cut off from the illumination optics. By further increasing the illumination angle, it is possible to achieve a sufficient separation of the illumination path and projection path, which at the same time leads to a further reduction of light throughput in the system.

The discussed advantages and disadvantages of the respective system architectures dependent on the technical implementation and the introduced illumination concepts leads to the following conclusion. The implementation of an imaging illumination of the active modulator area using a prism cannot be implemented with every illumination concept. In addition to that, the resulting defocus as well as the magnification of the images of the emission surfaces must be considered in the system design. Regardless of the illumination concept, the application of an LED array as a light source to illuminate the light modulator when implementing a non-telecentric system 
architecture by increasing the illumination angle can result in an insufficient separation of the illumination path and projection path.

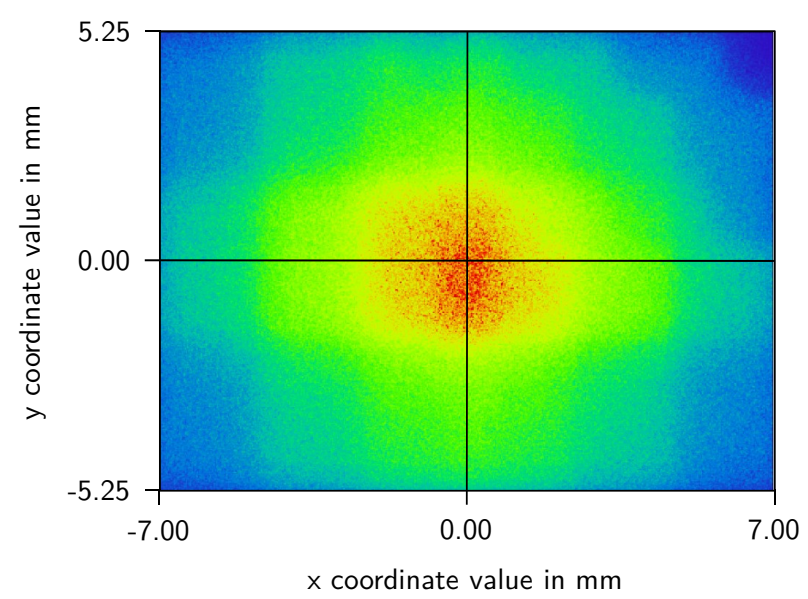

(a) Peak illuminance: $3.55 \cdot 10^{7} \frac{\mathrm{lm}}{\mathrm{m}^{2}}$

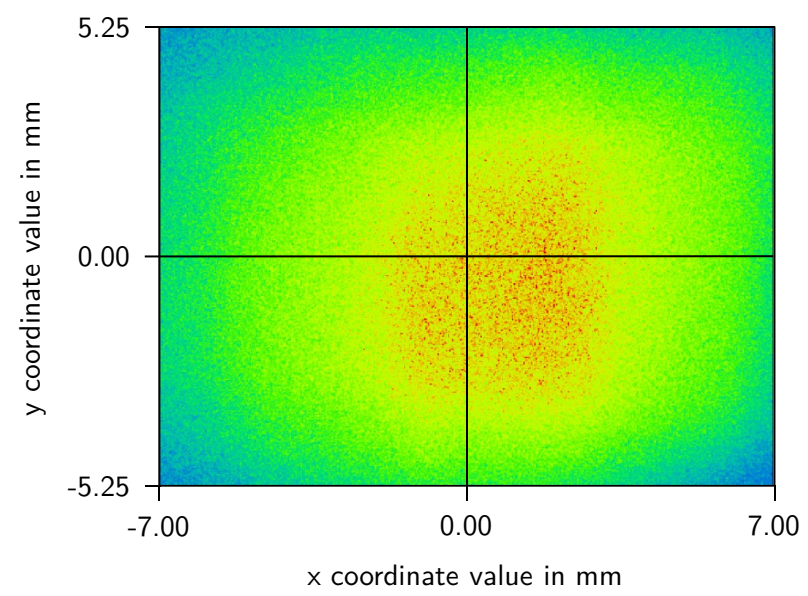

(c) Peak illuminance: $1.45 \cdot 10^{7} \frac{\mathrm{lm}}{\mathrm{m}^{2}}$

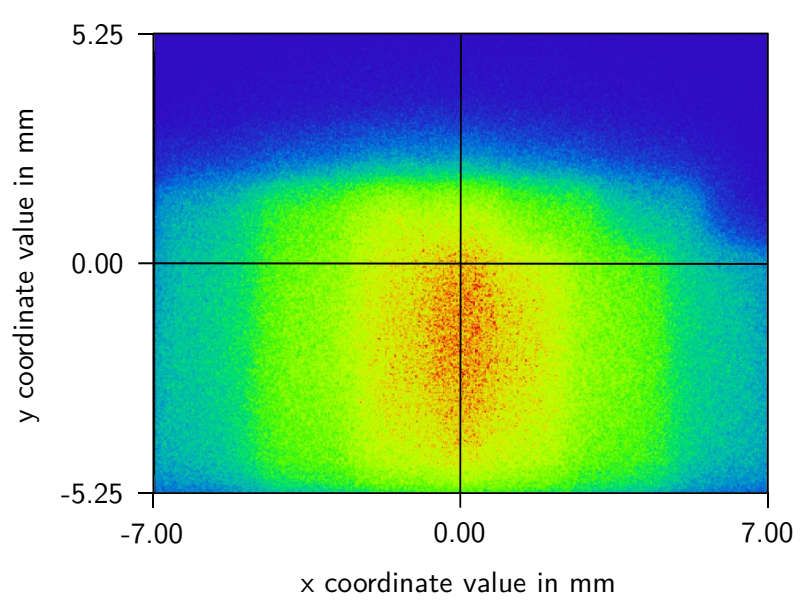

(b) Peak illuminance: $1.99 \cdot 10^{7} \frac{\mathrm{lm}}{\mathrm{m}^{2}}$

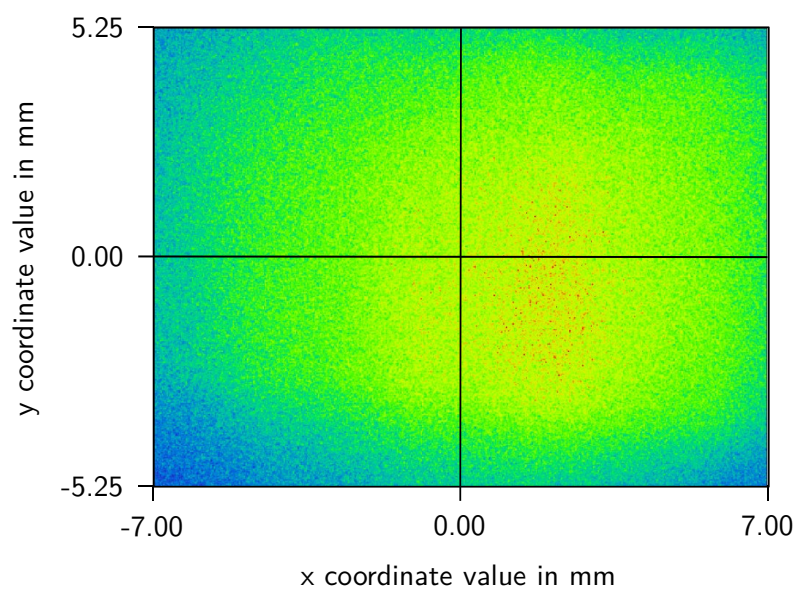

(d) Peak illuminance: $0,89 \cdot 10^{7} \frac{\mathrm{lm}}{\mathrm{m}^{2}}$

Figure 5. Imaging illumination of the active area of the modulator with all LEDs on (left side) and one row of the LED array switched off (right side); without TIR Prism: (a),(b); with TIR prism (c),(d)

The application of freeform mirrors and folding mirrors allow to implement a non-telecentric system architecture using a LED array as light source. However, this leads to a complicated and longer setup of the optical system. ${ }^{2}$ Therefore in this example a non-imaging inhomogeneous illumination of the active area of the modulator in combination with a telecentric system architecture by means of a TIR prism using a 2 x 4 LED array as a light source to implement a high-resolution headlamp will be used.

\section{TECHNICAL IMPLEMENTATION OF A NON-IMAGING INHOMOGENEOUS ILLUMINATION FOR A DLP IN A HIGH-RESOLUTION HEADLAMP}

One approach to implement an optical system for a high-resolution headlamp to illuminate the DLP and project it into the traffic area is shown by the numerical model in Figure 6. For the illumination of the DLP an optical system (green) consisting of three lenses is applied. Through a lens array, the light emitted by the eight LEDs is 
collimated and focused using the subsequent condenser lens. The third lens, which is not rotationally symmetric, allows the adjustment of the incident rays from the condenser lens. In combination with the following TIR prism, the rays are guided onto the active area of the DLP at the required angles and as uniform as possible with simultaneously minor overfill.

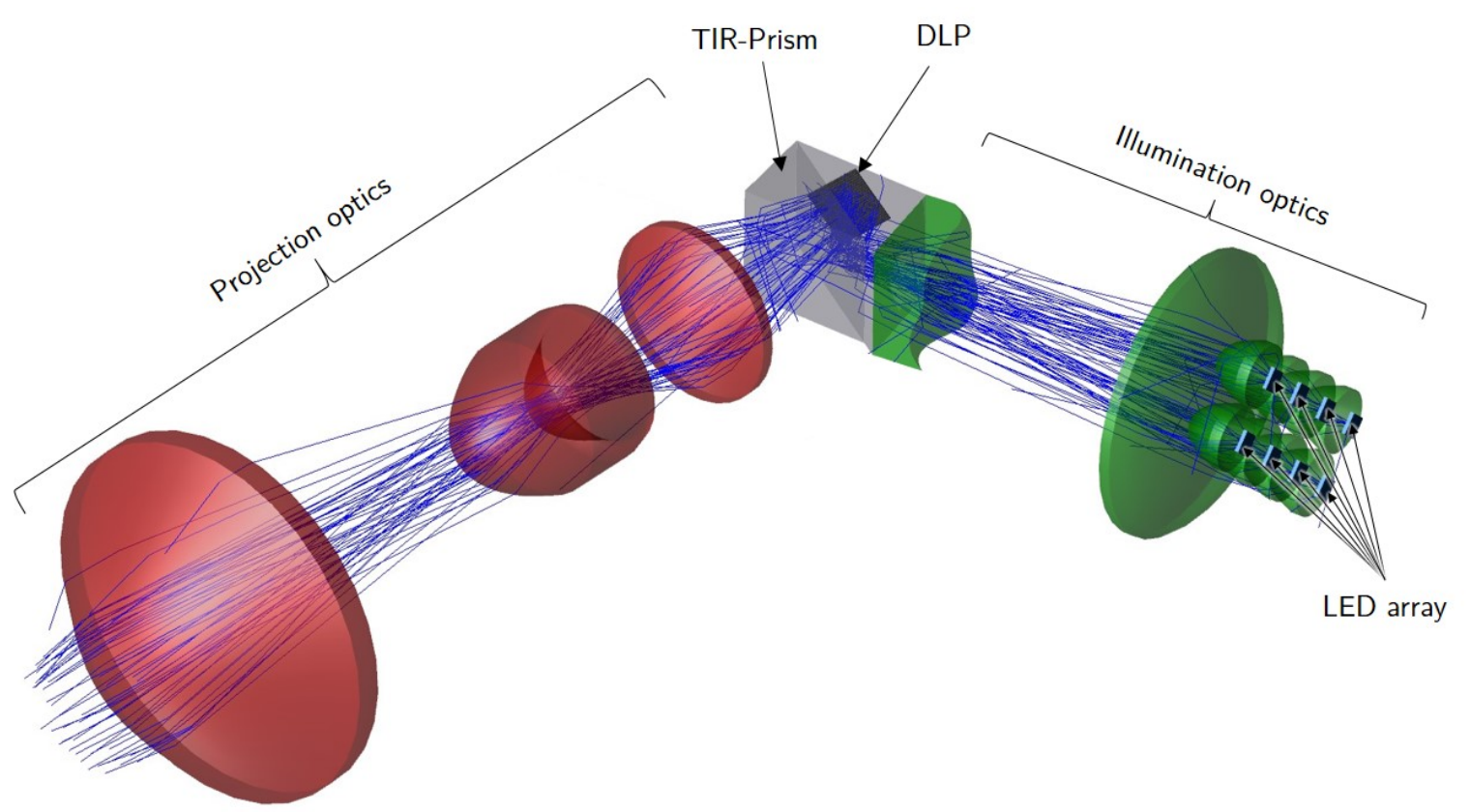

Figure 6. Numerical model of a DLP-based headlamp using an LED array consisting of 8 LEDs.

The desired non-imaging inhomogeneous illumination of the active DLP area to increase system efficiency can be seen in Figure 7. The distribution of illuminance corresponds to the concept of a non-imaging inhomogeneous illumination of the active area of the modulator presented in Table 2. Since a non-rotationally symmetrical lens is used for the third lens, the illuminance on the detector surface respectivley modulator surface has an elliptical distribution. This allows to keep a minor overfill.

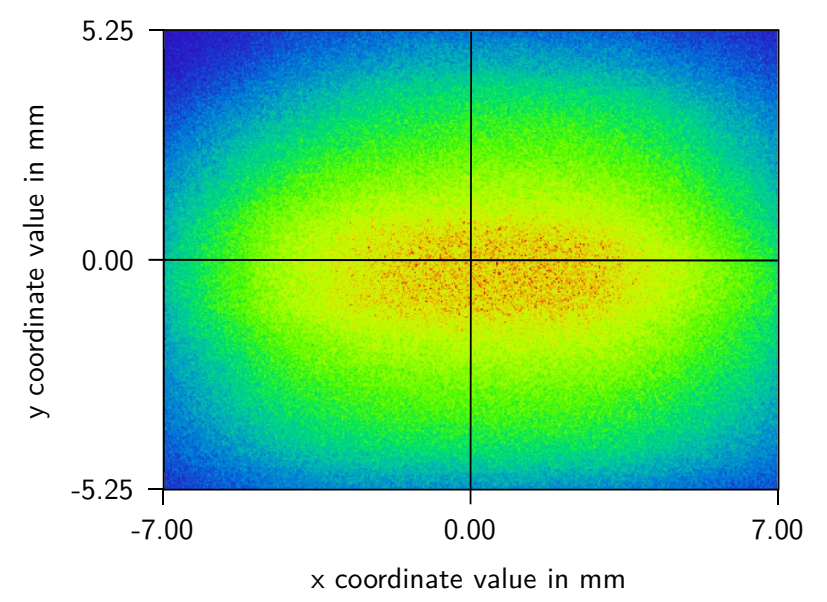

Figure 7. Non-Imaging inhomogeneous illumination of the active area of the modulator with all 8 LEDs on; Peak illuminance: $2,34 \cdot 10^{7} \frac{\mathrm{lm}}{\mathrm{m}^{2}}$ 
The required aspect ratio of 4:1 for the light distribution of the high-resolution headlamp necessitates an adaption of the aspect ratio of the DLP (4:3). This adaption can be implemented with various concepts of distortion for the projection optics, e.g. an anamorphic distortion. In this case we use an imaging optics (red) with a pincushion distortion which allows the adaption of the aspect ratio and a redistribution of the luminous flux after the DLP. The pincushion distortion of the projection optics leads to smaller pixels in the center with higher illuminance and larger pixels with reduced illuminance at the edge of the projected image..$^{8,9}$

\section{CONCLUSION}

Spatial light modulators are commonly used for entertainment in video projectors, but find more and more applications in other areas as well as in vehicle lighting technology. The use of spatial light modulators in vehicle headlamps enables the projection of information into the traffic area, due to the high amount of pixels, which can increase traffic safety. A promising technology is the DLP technology. Unlike other light modulators, such as LCD or LCoS, the DLP has the advantage to be able to modulate unpolarized light when being illuminated. However, all technologies have in common that they are subtractive imagers in which unused light is either reflected in an absorber (DLP) or blocked by liquid crystal (LCD or LCoS), thereby reducing the system efficiency. In the development of vehicle headlamps, efficiency is a crucial factor. Suitable concepts to illuminate the active area of the modulator allow to increase the system efficiency compared to a homogeneous illumination. In this paper we presented concepts for imaging and non-imaging illumination strategies of a DLP for highresolution headlamps. The presented concepts are discussed on a conceptual level regarding their suitability for high-resolution headlamps and their potential to increase the system efficiency compared to a homogeneous illumination depending on the system architecture and technical implementation.

In conclusion it can be stated that the implementation of an imaging illumination of the active modulator area using a TIR prism for a telecentric architecture cannot be implemented with every illumination concept. The TIR prism within the ray path leads to a magnification and defocus of the images of the emission surfaces, which has to be considered when designing the optical system. Regardless of the illumination concept, an implementation of a non-telecentric architecture by increasing the illumination angle of the DLP to separate the illumination path and projection path leads to a lower light throughput in the optical system. Additionally the separation of the two light paths can be insufficient when using a large light source such as an LED array. Whereas the application of freeform mirrors and folding mirrors to implement a non-telecentric system architecture enable the usage of an LED array as light source to illuminate the DLP but leads to a longer and more complicated setup of the optical system. Therefore a non-imaging inhomogeneous illumination of the active area of the modulator in combination with a telecentric architecture by means of TIR prism represents an interesting approach for the implementation of a high-resolution headlamp using an LED array as a light source. The telecentric system architecture allows a nearly independent design of the illumination optics and projection optics. By using a TIR prism to separate the two light paths, it is possible to achieve a sufficiently large separation of the two paths, whereby a large light source such as an LED array can be applied to illuminate the DLP. The suitability of the individual components in the automotive sector such as the TIR prism or the applied DLP to implement a high-resolution headlamp was not discussed.

\section{REFERENCES}

[1] Gut, C., Rotscholl, I., and Neumann, C., "Theoretische leistungs- und effizienzanalyse laserbasierter pixellichtsysteme," in [6. VDI-Tagung Optische Technologien in der Fahrzeugtechnik // Optische Technologien in der Fahrzeugtechnik], VDI, ed., VDI-Berichte 2221, 15-29, VDI-Verl., Düsseldorf (2014).

[2] Texas Instruments Incorporated, "Dlp system optics (application report no. dlpa022july 2010)," (2010). 
[3] Bhakta, V. R. and Ballard, B., "High resolution adaptive headlight using texas instruments dlp technology," in [11th International Symposium on Automotive Lighting], GmbH, H. U. V., ed., Darmstaedter Lichttechnik 16, 483-494 (2015).

[4] Ley, P.-P., Held, M. P., Wolf, A., and Lachmayer, R., "Konzepte zur beleuchtung von lichtmodulatoren," DGaO Proceedings (2018).

[5] Schott AG, "Optical glas datasheets," (2010).

[6] Texas Instruments Incorporated, DLP7000 DLP 0.7 XGA 2x LVDS Type A DMD (8 2012). Revised May 2017.

[7] Ley, P.-P., Held, M. P., and Lachmayer, R., "Analysis of led arrangement in an array with respect to lens geometry," in [Light-Emitting Diodes: Materials, Devices, and Applications for Solid State Lighting XXII], Novak, M., ed., Proc. SPIE 10554 (2018).

[8] Wolf, A., Kloppenburg, G., Danov, R., and Lachmayer, R., "Dmd based automotive lighting unit," DGaO Proceedings (2016).

[9] Wolf, A. G., Lachmayer, R. J., and Kloppenburg, G., "Lighting device, lighting method and computer program," (Sep 2017). 